[Original]

\title{
Effect of Psychological Distress on the Association of Workplace Social Capital with Presenteeism and Sickness Absence
}

\author{
Miho OMOri $^{1 *}$, Tomohisa Nagata ${ }^{2}$, Masako Nagata $^{2}$, Yoshihisa Fujino ${ }^{3}$ and Koji Mori ${ }^{2}$ \\ ${ }^{1}$ Department of Occupational and Community Health Nursing, School of Health Sciences, University of Occupational \\ and Environmental Health, Japan. Yahatanishi-ku, Kitakyushu 807-8555, Japan \\ ${ }^{2}$ Department of Occupational Health Practice and Management, Institute of Industrial Ecological Sciences, \\ University of Occupational and Environmental Health, Japan. Yahatanishi-ku, Kitakyushu 807-8555, Japan \\ ${ }^{3}$ Department of Environmental Epidemiology, Institute of Industrial Ecological Sciences, University of Occupational \\ and Environmental Health, Japan. Yahatanishi-ku, Kitakyushu 807-8555, Japan
}

\begin{abstract}
Greater workplace social capital (WSC) can be related to workers' health and productivity. We sought to clarify the association between horizontal WSC and presenteeism and sickness absence (SA) and to examine the effects of psychological distress on these associations among Japanese workers. A cross-sectional study was conducted in 2017 at seven large Japanese companies. Logistic regression analysis was performed with presenteeism and SA as the dependent variables, horizontal WSC as an independent variable, and sociodemographic characteristics and psychological distress as covariates. After adjustment for sociodemographic characteristics, the results showed that greater horizontal WSC was associated with lower presenteeism and SA. The odds ratios for the relationship between horizontal WSC and presenteeism and that between horizontal WSC and SA dropped moderately after adjustment for psychological distress but remained significant. Further exploration of the factors underlying the relationship between WSC and productivity is needed to confirm if WSC enhances workers' health and productivity and to inform the development of effective occupational health initiatives.
\end{abstract}

Keywords : workplace social capital, presenteeism, sickness absence, psychological distress.

(Received October 27, 2020, accepted April 2, 2021)

\section{Introduction}

Social capital is characterized by social organizations such as networks, norms, and trust that promote action and cooperation for mutual benefit [1]. The relationships between social capital and health-related outcomes, such as self-rated health, risk and symptoms of mental disorders, and mortality, have been studied in the field of public health for at least 20 years $[2,3]$. The workplace is a community in which people spend a substantial amount of time and interact with others through social networks [4]. In recent years, the workplace has been a target of social capital research, with several studies investigating the relationship between social capital and worker health and productivity [57]. Social capital in the workplace, termed workplace social capital (WSC), has been defined as "mutual trust, justice and collaboration in the workplace" and is associated with aspects of psychosocial relationships such as cooperation in the workplace [8-11].

*Corresponding Author: Miho Omori, Department of Occupational and Community Health Nursing, school of Health Sciences, University of Occupational and Environmental Health, Japan. 1-1 Iseigaoka, Yahatanishi-ku, Kitakyushu 807-8555, Japan. Tel: +81-93-691-7160, Fax: +81-93-692-0259, E-mail: omorimiho@health.uoeh-u.ac.jp 
WSC is regarded as a resource for worker health and health-promoting activities, and previous studies indicate that it can determine worker health $[12,13]$. Rich WSC is characterized by social support, mutual trust, and constructive cooperation between workers and between workers and their supervisors [11]. When workers in high WSC workplaces encounter stressful situations, for example, trust develops between workers and their superiors and colleagues, facilitating the utilization of resources such as support and mental health care [14]. This promotes the exchange of information and emotional support from colleagues, which can assist in health-related changes [15]. Workplaces with high WSC, such as those with well-established norms, are more likely to encourage desirable healthy behaviors $[16,17]$.

WSC is associated with several lifestyle diseases, including hypertension [6, 18, 19], obesity [18, 19] and diabetes [19], health-impairing habits such as smoking [20] and drinking [21], positive psychological states such as job satisfaction $[22,23]$ and work engagement $[7,23]$, and other health-related factors such as wellbeing [7] and self-rated health [5]. Lower WSC has adverse effects on workers' mental health, and is associated with mental health problems such as depression and psychological distress [17, 24, 25].

Workplaces are characterized by several relationship structures, including hierarchical relationships among management, supervisors, and colleagues, as well as relationships among colleagues in the same department and other departments. WSC can therefore be categorized in terms of horizontal and vertical indicators [26]. Horizontal WSC includes bonding, which involves social relationships between individuals with similar socioeconomic attributes (e.g., colleagues in the same department), and bridging, which represents relationships between persons with different attributes at the same hierarchical level (e.g., interdepartmental relationships); vertical WSC includes relationships between persons across hierarchical levels [26-28]. In a survey of 37 countries, the International Social Survey Program reported that Japan had the lowest percentage of people who thought that "in my workplace, the relationships between colleagues in the workplace are good," which indicates that horizontal WSC in the Japanese workplace is poor [29]. There is also evi- dence that relationships with colleagues affect health. For example, coworker support contributes to women's health [30], lack of coworker trust and assistance is associated with high systolic blood pressure [31], and poor coworker trust (i.e., poor horizontal WSC) is associated with worker health problems [32]. These findings suggest that horizontal WSC, which is characterized by relationships with colleagues in the workplace, has substantial effects on health.

The WSC factor of workplace collaboration can affect worker health and the efficiency of production processes [7]. The loss of productivity from health problems is often assessed in terms of presenteeism and sickness absenteeism [33-35]. The term presenteeism is commonly used to describe two different concepts: going to work despite feeling unhealthy, and productivity loss at work owing to health problems $[36,37]$. Studies in both the United States and Japan have reported substantial productivity loss from presenteeism $[35,38]$. Presenteeism related to productivity loss includes lifestyle diseases and physical symptoms [35, 39-43], but the most important health issues are mental health-related problems in the workplace, such as stress, psychological distress, and depression [43-46]. Studies in Japan have indicated that mental and behavioral disorders are the most common causes of presenteeism [35]. In contrast to presenteeism, sickness absence (SA) describes absence from work caused by sickness and can be either short term or long term [47]. The main causes of SA are mental health problems, such as depression, and musculoskeletal disorders $[48,49]$.

The deterioration of worker physical and mental health increases presenteeism and SA, and reduces the productivity of companies. The improvement of WSC could possibly reduce presenteeism and SA, but few studies have examined the relationships between WSC and presenteeism and SA, and whether health factors affect these relationships. To the best of our knowledge, only two previous studies have examined the relationship between WSC and presenteeism, and a negative dose-response relationship was observed $[50,51]$. Several previous studies have examined the relationship between WSC and SA, but the results are inconsistent; although multiple studies have found an association between higher WSC and lower SA [52- 
54], another study reported a non-significant relationship between WSC and SA [55]. Although Japanese workers are presumed to have poor horizontal WSC, the relationship between horizontal WSC and presenteeism (which may affect health) and between horizontal WSC and SA in Japanese workers remains to be clarified.

As mentioned above, a significant relationship between horizontal WSC and health status has been established, and health status has been shown to affect presenteeism and SA. Thus, it is necessary to investigate whether health status affects the relationship between horizontal WSC and presenteeism, and that between horizontal WSC and SA. Previous studies have shown that subjective health and job stress influence the relationship between WSC and presenteeism [50, 51], but the effects of mental health on the relationship between WSC and presenteeism, and on that between WSC and SA, have not been examined. As WSC is an important resource for the promotion of mental health and can reduce stress [55], high WSC might be expected to reduce presenteeism and SA that result from mental health problems. Among indicators of mental health, Kessler's six-item psychological distress scale (K6) is a reliable and valid measure of mental health status [56].

This study aimed to 1) elucidate the relationship between horizontal WSC and presenteeism and the relationship between horizontal WSC and SA among Japanese employees, and 2) determine the effects of psychological distress on these relationships.

\section{Methods}

\section{Study population and data collection}

This was a cross-sectional study. Participants were workers older than 20 years of age from seven large Japanese companies: five manufacturing companies and two service companies. Two of the companies were listed in the First Section of the Tokyo Stock Exchange. A self-administered website survey was administered to 33,980 workers between July 2017 and October 2017. The number of participants in the seven companies were as follows: A: $n=897 ; \mathrm{B}: \mathrm{n}=2,287$; $C: n=3,116 ; D: n=5,475 ; E: n=6,066 ; F: n=7,040$; $\mathrm{G}: \mathrm{n}=9,099$.

\section{Workplace social capital (explanatory variable)}

Previous studies have assessed WSC in different ways, but WSC measures are typically based on self-administered questionnaires, and various indicators have been developed [27]. Items from different scales have been used in combination to measure the multifaceted elements of WSC, or authors have added new items to existing WSC measures [23]. In the present study, we used the Japanese version of the WSC scale, which is based on a scale by Kouvonen et al $[27,57]$, and the WSC scale developed by Tsuboya et al for Japanese workers [25], to evaluate horizontal WSC. The Kouvonen et al WSC scale comprises eight items, three of which are vertical items and five of which are horizontal items [27, 28]. In the present study, we used the following five horizontal items: (1) We have a "we are together" attitude; (2) People keep each other informed about work-related issues in the work unit; (3) People feel understood and accepted by each other; (4) Members of the work unit build on each other's ideas to achieve the best possible outcome; (5) People in the work unit cooperate to help develop and apply new ideas. We added to that the two horizontal questions from the six WSC scale items developed by Tsuboya et al [25]; (6) In our workplace, we trust each other; (7) Our workplace is a place of laughter and smiles. These seven questions had four response options and were scored: 1$)$ strongly agree (4 points), 2) agree (3 points), 3) disagree ( 2 points), and 4 ) strongly disagree (1 point).

The WSC scale developed by Tsuboya et al is based on the WSC scale developed by Kouvonen et al, and items (6) and (7) above are newly created items that were added to the present survey because they reflect the characteristics of Japanese companies. The horizontal WSC score comprises the total score across the seven items, with a score range from 7 to 28 ; higher scores indicated greater horizontal WSC. Because there is no standard WSC cutoff point, the distribution of scores was divided into three quartile categories: low (7-19 points), moderate (20-22 points), and high (23-28 points). Cronbach's alpha for horizontal WSC scores was 0.93 in this study.

WSC has been measured at both the individual level and the workplace level, but the best measurement method remains to be determined; in this study, hori- 
zontal WSC was measured at the individual level for the following reason. WSC is a characteristic of individuals, and the association between WSC and health has been clarified by individual-level analysis. A group-level analysis can be conducted using the average value of social capital in the workplace, but grouplevel WSC is generally weakly related to or unrelated to health, so individual-level analysis is recommended for health-based WSC studies [55].

\section{Outcomes}

The outcomes were that a higher score indicates moderate or greater work function impairment (presenteeism) and SA. Presenteeism was measured by the Work Functioning Impairment Scale (WFun) [58], which was developed to measure the level of work impairment in workers based on the Rasch model. It is a self-assessment scale comprising seven items: (1) I haven't been able to behave socially; (2) I haven't been able to maintain the quality of my work; (3) I have had trouble thinking clearly; (4) I have taken more breaks during my work; (5) I have felt that my work isn't going well; (6) I haven't been able to make rational decisions; and (7) I haven't been proactive about my work. Each item has five response options: 1) not at all, 2) at least 1 day a month, 3) about 1 day a week, 4) at least 2 days a week, and 5) almost every day. The WFun score comprises the total score across seven items, with a score range of 7 to 35 . Higher scores indicate greater impairment in work functioning. A score of 21 or more indicates moderate or greater work function impairment. In terms of validity, previous studies have reported that moderate or greater work impairment on this scale is strongly associated with independent assessments conducted by an occupational health nurse regarding the extent to which health problems affect work [58]. We defined a high WFun score as 21 points or more, and a low score as below 21 points.

SA was measured using the following question: "Please indicate the number of days you have missed work due to your own medical treatment in the past year." The number of days off was a continuous response variable. Human resource data kept by employers is the most accurate measure of SA [59], but previous research has also used self-report measures, which indicates that alternative SA measures are fea- sible [60]. As there is no standard cutoff point for annual absences, we calculated the mean number of days of sick leave of subjects with SA (mean 5.1, standard deviation [SD] 14.2, median 2.4) and used 5 days as the cutoff point.

\section{Covariates}

Sociodemographic characteristics (sex, age category, company, occupation, and employment position) and psychological distress were included as covariates.

Personnel data from each company were used for sex and age. For age, continuous variable data were categorized into 20-29 years, 30-39 years, 40-49 years, 50-59 years, and 60 years and above. Respondents were asked to choose from the following occupational categories: office/staff, sales, customer service, research, development, technical/skilled worker, factory production line, production technician, and other. Psychological distress was evaluated using the Japanese version of the K6, which comprises six items that measure the degree of psychological distress [56]. Total scores range from 0 to 24; higher scores indicate more psychological distress. Scores of 5 or more are considered to indicate mild or greater psychological distress. A cutoff score of 5 or more points was considered an appropriate indicator of mild or greater psychological distress for our survey of Japanese respondents [61, 62].

\section{Statistical analysis}

First, a chi-square test was used to compare the ratios of horizontal WSC high, intermediate, and low groups. Next, using horizontal WSC as the explanatory variable and presenteeism and SA as the dependent variables, a multiple logistic regression analysis was performed to identify the relationship between horizontal WSC and presenteeism, the relationship between horizontal WSC and SA, and odds ratios (OR) and 95\% confidence intervals (CI) for both relationships. We adjusted for sex and age categories (Model 1), then added company, occupation, and job position (Model 2). Finally, we adjusted for K6 score (Model 3). The significance level was set at two-sided $P<0.05$, and SPSS version 25.0 (IBM Corp., Armonk, NY) was used for statistical analysis. 


\section{Ethics}

The purpose of the study was explained to the participants, and their consent to participate was obtained before the survey was conducted. Workers were free to choose whether to participate in the study. The results from the self-administered questionnaires completed by workers were not made available to the companies. The research protocol was approved by the medical research ethics committee of the University of Occupational and Environmental Health, Japan (H26-026).

\section{Results}

There were 17,532 respondents; the response rate per company ranged from $12 \%$ to $81 \%$. After excluding 3,376 individuals for whom data were missing, the data of 14,156 individuals were analyzed.

The characteristics of the 14,156 subjects are shown in Table 1, in which $77 \%(\mathrm{n}=10,852)$ were men. The mean (SD) age was 43.9 years (9.86) (data not shown in table). In terms of occupation, $64 \%$ of the subjects were sales and office/staff, and $22 \%$ had the job position of manager. A total of $30 \%(n=4,261)$ had high K6 scores, $32 \%(n=4,569)$ were in the low horizontal WSC group, $38 \%(n=5,428)$ were in the intermediate horizontal WSC group, and 30\% $(\mathrm{n}=4,159)$ were in the high horizontal WSC group. The mean K6 score was $3.52(\mathrm{SD}=4.38)$ and the mean horizontal WSC score was $21.3(\mathrm{SD}=3.95)$ (data not shown in table). Fourteen percent $(\mathrm{n}=1,922)$ of the subjects had presenteeism and $14 \%(n=1,977)$ had SA of 5 days or more. Significant differences in the proportion of horizontal WSC degree were found by sex. Similar significant differences were also found for factors such as age categories, occupation, job position, K6 score, presenteeism and SA $(P<0.001)$.

Table 2 shows the results of multiple logistic regression analysis of the relationship between horizontal WSC and presenteeism. In Model 2, which was adjusted for sex, age categories, company, occupation, and job position, lower presenteeism was associated with greater horizontal WSC (adjusted OR: 0.48 [CI: 0.43-0.54] for intermediate horizontal WSC; adjusted OR 0.27 [CI: 0.24-0.31] for high horizontal WSC). In Model 3, this relationship remained significant when the K6 score was included in Model 2 as a covariate (adjusted OR: 0.66 [CI: 0.58-0.74] for intermediate horizontal WSC; adjusted OR 0.47 [CI: 0.41-0.55] for high horizontal WSC). The $P$ value of the linear tendency was statistically significant in Model 1 to Model 3. Table 3 shows the results of multiple logistic regression analysis of the relationship between horizontal WSC and SA. In Model 2, which was adjusted for sex, age categories, company, occupation, and job position, lower SA was associated with greater horizontal WSC (adjusted OR: 0.76 [CI: 0.68-0.84] for intermediate horizontal WSC; adjusted OR: 0.65 [CI: 0.58-0.74] for high horizontal WSC). In Model 3, this relationship remained significant when the K6 score was included in Model 2 as a covariate (adjusted OR: 0.82 [CI: 0.73-0.92] for intermediate horizontal WSC; adjusted OR: 0.74 [CI: 0.65-0.85] for high horizontal WSC). The $P$ value of the linear tendency was statistically significant in Model 1 to Model 3.

\section{Discussion}

The present findings showed that, in Japanese companies, greater horizontal WSC, analyzed at the individual-level after adjusting for sociodemographic characteristics, was associated with lower presenteeism (measured as work functional impairment) and lower SA. The present finding that a lower risk of presenteeism was associated with greater WSC is consistent with a previous study by van Scheppingen et al, which found that horizontal WSC (bonding WSC) was significantly negatively associated with presenteeism in a cross-sectional study of Dutch workers [51]. Zhu et al also conducted a cross-sectional study of workers in 38 different Chinese factories and reported that WSC, assessed by a combination of horizontal and vertical factors after adjusting for socioeconomic characteristics, was negatively associated with presenteeism at both the individual and workplace levels [50]. The present results also showed that greater horizontal WSC was associated with lower SA, in accord with previous studies in the Netherlands by van Scheppingen et al [51]. Van Scheppingen et al found a negative relationship between horizontal WSC (bonding WSC) and absenteeism, and Török et al found that higher levels of a combination of horizontal and vertical WSC factors in work units were associated with 
Table 1. Subject characteristics and descriptive data according to horizontal workplace social capital category

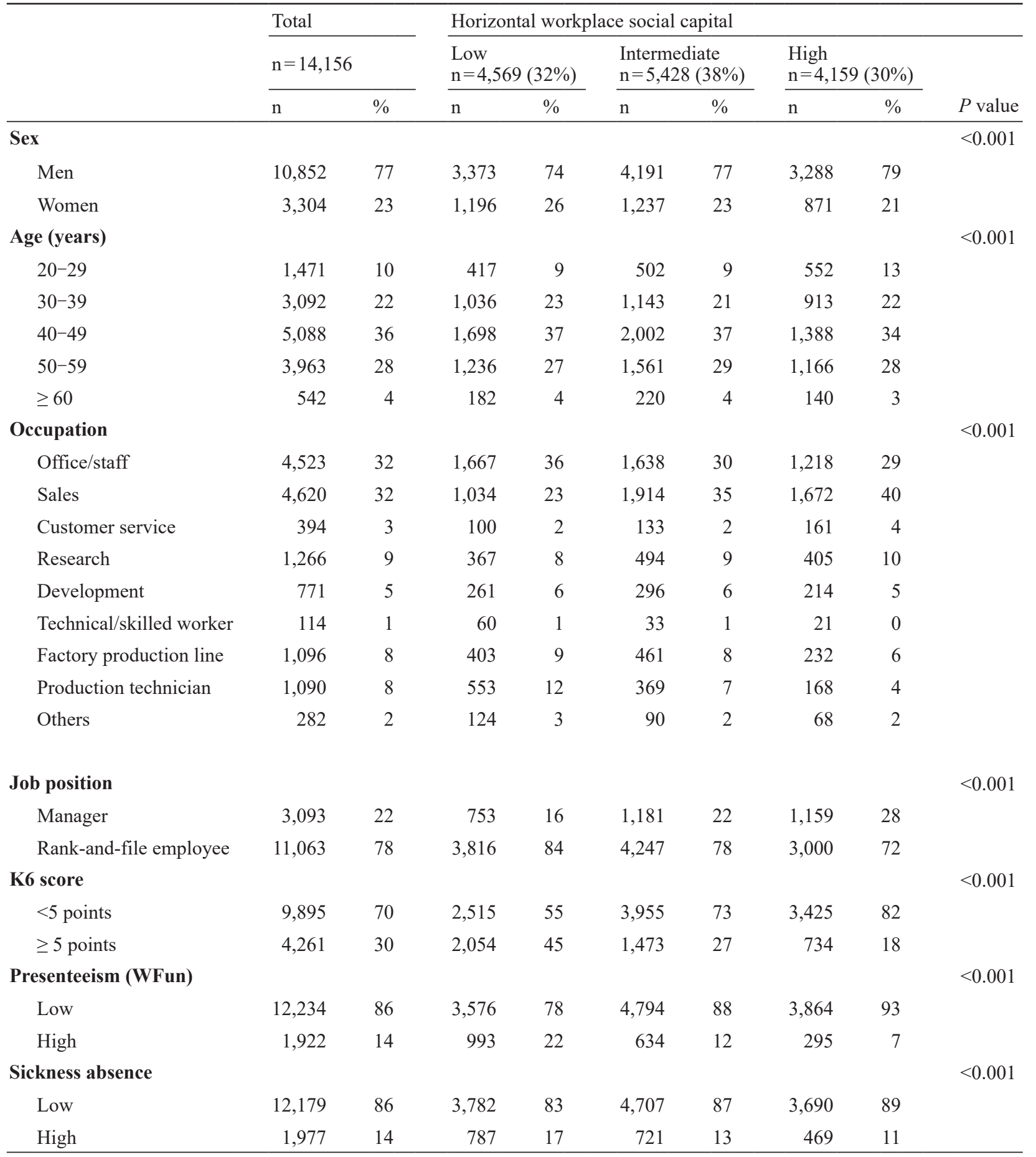

Presenteeism (WFun): Work Functioning Impairment Scale, sickness absence: number of days missed from work owing to medical treatment in the past year. 
Table 2. Odds ratios and $95 \%$ confidence intervals of multivariable logistic regression analysis of horizontal workplace social capital and presenteeism

\begin{tabular}{|c|c|c|c|c|c|c|c|c|c|}
\hline & \multicolumn{3}{|c|}{ Model 1} & \multicolumn{3}{|c|}{ Model 2} & \multicolumn{3}{|c|}{ Model 3} \\
\hline & $\mathrm{aOR}$ & $95 \% \mathrm{CI}$ & $P$ value & $\mathrm{aOR}$ & $95 \% \mathrm{CI}$ & $P$ value & $\mathrm{aOR}$ & $95 \% \mathrm{CI}$ & $P$ value \\
\hline \multicolumn{10}{|c|}{ Horizontal workplace social capital } \\
\hline Low & \multicolumn{3}{|c|}{ reference } & \multicolumn{3}{|c|}{ reference } & \multicolumn{3}{|c|}{ reference } \\
\hline Intermediate & 0.47 & $0.43-0.53$ & $<0.001$ & 0.48 & $0.43-0.54$ & $<0.001$ & 0.66 & $0.58-0.74$ & $<0.001$ \\
\hline High & 0.27 & $0.23-0.31$ & $<0.001$ & 0.27 & $0.24-0.31$ & $<0.001$ & 0.47 & $0.41-0.55$ & $<0.001$ \\
\hline
\end{tabular}

aOR: adjusted odds ratio, CI: confidence interval, presenteeism (WFun): Work Functioning Impairment Scale. Model 1: Adjusted for sex, age category. Model 2: Adjusted for model $1+$ company, occupation, and job position. Model 3: Adjusted for model $2+$ K6 score.

Table 3. Odds ratios and $95 \%$ confidence intervals of multivariable logistic regression analysis of horizontal workplace social capital and sickness absence

\begin{tabular}{|c|c|c|c|c|c|c|c|c|c|}
\hline & \multicolumn{3}{|c|}{ Model 1} & \multicolumn{3}{|c|}{ Model 2} & \multicolumn{3}{|c|}{ Model 3} \\
\hline & $\mathrm{aOR}$ & $95 \% \mathrm{CI}$ & $P$ value & $\mathrm{aOR}$ & $95 \% \mathrm{CI}$ & $P$ value & $\mathrm{aOR}$ & $95 \% \mathrm{CI}$ & $P$ value \\
\hline \multicolumn{10}{|c|}{ Horizontal workplace social capital } \\
\hline Low & \multicolumn{3}{|c|}{ reference } & \multicolumn{3}{|c|}{ reference } & \multicolumn{3}{|c|}{ reference } \\
\hline Intermediate & 0.74 & $0.67-0.83$ & $<0.001$ & 0.76 & $0.68-0.84$ & $<0.001$ & 0.82 & $0.73-0.92$ & $<0.001$ \\
\hline High & 0.62 & $0.55-0.71$ & $<0.001$ & 0.65 & $0.58-0.74$ & $<0.001$ & 0.74 & $0.65-0.85$ & $<0.001$ \\
\hline
\end{tabular}

aOR: adjusted odds ratio, CI: confidence interval, sickness absence: number of days missed from work owing to medical treatment in the past year. Model 1: Adjusted for sex, age category. Model 2: Adjusted for model $1+$ company, occupation, and job position. Model 3: Adjusted for model $2+$ K6 score.

lower long-term SA [52].

Rich WSC may help to develop a healthy code of conduct in the workplace and to promote the rapid dissemination of useful health information [52]. Health behavior is better in workplaces in which health norms are high and information about health is actively exchanged, which is likely to improve worker performance and reduce presenteeism. High WSC workplaces have established norms and trust, so physical work tasks can be distributed more efficiently and the health status of individual workers can be addressed [52]. Workplaces with high WSC can therefore be characterized by low SA. These previous studies suggest that WSC is an important resource that affects worker productivity, and the present findings indicate a relationship between rich WSC and lower presenteeism and SA.

It has been suggested that rich WSC is characterized by higher health norms, that good workplace cohesiveness promotes healthy behavior, and that a sense of trust reduces stress $[16,17]$. Thus, high WSC may lower presenteeism and SA. The present findings showed that after adjustment for psychological distress, the ORs for the relationship between horizontal WSC and presenteeism, and for that between horizontal WSC and SA, dropped moderately but remained significant. As mentioned above, previous study findings differ regarding the effects of health on presenteeism and SA.

To the best of our knowledge, only one previous study has adjusted for health indicators in the relationship between WSC and presenteeism and SA: a study of workers in the Netherlands by van Scheppingen et al [51]. In the study, the negative relationships of presenteeism and absenteeism with a combination of horizontal and vertical factors WSC, respectively, were no longer significant when self-rated health and emotional exhaustion were included in the regression model.

Thus, although we used different health indicators, it is clear that the one previous study mentioned above and the present study yielded different results regarding the effects of health conditions. There are several possible reasons for these contrasting results. First, differences in WSC measures may have contributed to 
the discrepancies. Whereas the study by van Scheppingen et al measured a combination of horizontal and vertical factors of WSC in the workplace [51], the present study examined only horizontal WSC. Vertical WSC is characterized by the formation of reliable and respectful relationships, and reduces stress related to a lack of power and control over the job [63], but this aspect was not included in the present study. The second possible reason is related to differences in the health indicators used. Van Scheppingen et al examined self-rated health and emotional fatigue, which is a subscale on the Utrecht Burnout Scale [51]. In the present study, however, K6 was used as a health indicator. Third, differences in the target population may also have affected the results. However, the present findings suggest that factors other than psychological distress affected the relationship between horizontal WSC and presenteeism, and that between horizontal WSC and SA.

\section{Limitations}

The present study had several limitations that should be considered. First, as the data were cross-sectional, it is not possible to draw firm conclusions about the causal relationships between horizontal WSC and presenteeism and SA. Longitudinal studies are needed to determine any causal relationships.

Second, there were several limitations regarding the measurement of WSC. We used individual-level WSC because organizational information about each company was not available, but Clausen et al reported that higher levels of uniformity were found between the results of individual- and team-level analyses [7]. In contrast, Meng et al reported that WSC is measured most appropriately at the level of work teams in the workplace because work teams are the social units that are most accessible for cooperation and social support [16]. Another limitation is that the present study only measured horizontal WSC and so was unable to assess the effect of WSC at the vertical level. Although some previous studies have measured horizontal WSC and vertical WSC separately, it cannot be assumed that they provide a coherent measure of horizontal and vertical WSC aspects.

Third, there were several limitations regarding data quality. The data were self-reported and vulnerable to response and recall bias. In addition to that, we recorded the number of days of absence owing to illness in the past year, which resulted in temporal discrepancies with variables such as horizontal WSC at the time of the survey. Although previous SA is associated with current social support [64], the possibility that the time gap had an effect cannot be ruled out.

Fourth, SA was calculated using the average number of absence days per year, which may not differentiate between short-term and long-term SA, potentially making the results vulnerable to bias toward long-term absence.

Fifth, the questionnaire response rate varied between companies because the methods used to request survey cooperation differed between them. We conducted an analysis that included companies as covariates, but could not completely rule out that effect.

Sixth, participants were workers in the manufacturing and service sectors of large companies, and the findings may not be generalizable to workers in small and medium companies and other industries.

Despite these limitations, the strengths of the study are the large sample size and the robust results for the relationship between horizontal WSC and presenteeism and SA.

\section{Conclusion}

This study showed that greater horizontal WSC was associated with lower risk of presenteeism and SA in Japanese companies. These findings suggest that horizontal WSC may promote workers' health and productivity, and that efforts to improve WSC should be undertaken. However, many of these factors and mechanisms remain unclear. Further exploration of the factors underlying the association between WSC and productivity is needed, including intervention studies. Such work could inform approaches for enhancing WSC by developing occupational health activities that contribute to workers' health and productivity.

\section{Acknowledgements}

This study was supported by the Collabo-Health Study Group. 


\section{Conflict of Interest}

The authors declare that they have no conflict of interest.

\section{References}

1. Putnam RD, Leonardi L \& Nanetti RY (1993): Making Democracy Work: Civic Traditions in Modern Italy. Princeton University Press, Princeton 257pp

2. Rodgers J, Valuev AV, Hswen Y \& Subramanian SV (2019): Social capital and physical health: an updated review of the literature for 2007-2018. Soc Sci Med 236: 112360

3. Rotenberg M, Anderson KK \& McKenzie K (2020): Social capital and psychosis: a scoping review. Soc Psychiatry Psychiatr Epidemiol 55(6): 659-671

4. Lindström M (2008): Invited commentary: social capital, social contexts, and depression. Am J Epidemiol 167(10): 1152-1154

5. Han S, Park KK \& Lee JW (2020): Does organization matter for health? The association between workplace social capital and self-rated health. J Occup Environ Med 62(5): 331-336

6. Oksanen T, Kawachi I, Jokela M et al (2012): Workplace social capital and risk of chronic and severe hypertension: a cohort study. J Hypertens 30(6): 1129-1136

7. Clausen T, Meng A \& Borg V (2019): Does social capital in the workplace predict job performance, work engagement, and psychological well-being? A prospective analysis. J Occup Environ Med 61(10): 800-805

8. Oksanen T, Kivimaki M, Kawachi I et al (2011): Workplace social capital and all-cause mortality: a prospective cohort study of 28,043 public-sector employees in Finland. Am J Public Health 101(9): 1742-1748

9. Jensen JH, Flachs EM, Skakon J, Rod NH \& Bonde JP (2019): Longitudinal associations between organizational change, work-unit social capital, and employee exit from the work unit among public healthcare workers: a mediation analysis. Scand J Work Environ Health 45(1): 53-62

10. Hvidtfeldt UA, Bjorner JB, Jensen JH et al (2017): Cohort profile: the Well-being in HospitAL Employees (WHALE) study. Int J Epidemiol 46(6): 1758-1759h

11. Meng A, Borg V \& Clausen T (2019): Enhancing the social capital in industrial workplaces: developing workplace interventions using intervention mapping. Eval Program Plann 72: 227-236

12. Oksanen T, Suzuki S, Takao S, Vahtera J \& Kiviak M (2013): Workplace social capital and health. In: Global Perspectives on Social Capital and Health. (Kawachi I, Takao S \& Subramanian SV, ed). Nippon Hyoronsya, Tokyo pp22-63 (in Japanese)

13. Jung J, Ernstmann N, Nitzsche A et al (2012): Exploring the association between social capital and depressive symptoms: results of a survey in German information and communication technology companies. J Occup Environ Med 54(1): 23-30

14. Hori D, Takao S, Kawachi I et al (2019): Relationship between workplace social capital and suicidal ideation in the past year among employees in Japan: a crosssectional study. BMC Public Health 19(1): 919

15. Tsuboya T, Tsutsumi A \& Kawachi I (2016): Null association between workplace social capital and body mass index. Results from a four-wave panel survey among employees in Japan (J-HOPE study). Soc Sci Med 150: 1-7

16. Meng A, Clausen T \& Borg V (2018): The association between team-level social capital and individual-level work engagement: differences between subtypes of social capital and the impact of intra-team agreement. Scand J Psychol 59(2): 198-205

17. Gao J, Weaver SR, Dai J et al (2014): Workplace social capital and mental health among Chinese employees: a multi-level, cross-sectional study. PLoS One 9(1): e85005

18. Siervo M, Wells JC \& Stephan BC (2012): Workplace environment and risk of hypertension: is obesity on the causal pathway? J Hypertens 30(6): 1106-1107

19. Alterman T, Tsai R, Ju J \& Kelly KM (2019): Trust in the work environment and cardiovascular disease risk: findings from the Gallup-Sharecare Well-Being Index. Int J Environ Res Public Health 16(2): 230

20. Lindstrom M (2003): Social capital and the miniaturization of community among daily and intermittent smokers: a population-based study. Prev Med 36(2): 177-184

21. Gao J, Weaver SR, Fua H \& Pan Z (2014): Does workplace social capital associate with hazardous drinking among Chinese rural-urban migrant workers? PLoS One 9(12): e115286

22. Ommen O, Driller E, Kohler T et al (2009): The rela- 
tionship between social capital in hospitals and physician job satisfaction. BMC Health Serv Res 9: 81

23. Strömgren M, Eriksson A, Bergman D \& Dellve L (2016): Social capital among healthcare professionals: a prospective study of its importance for job satisfaction, work engagement and engagement in clinical improvements. Int J Nurs Stud 53: 116-125

24. Sakuraya A, Imamura K, Inoue A et al (2017): Workplace social capital and the onset of major depressive episode among workers in Japan: a 3-year prospective cohort study. J Epidemiol Community Health 71(6): 606-612

25. Tsuboya T, Tsutsumi A \& Kawachi I (2015): Change in psychological distress following change in workplace social capital: results from the panel surveys of the JHOPE study. Occup Environ Med 72(3): 188-194

26. Szreter S \& Woolcock M (2004): Health by association? Social capital, social theory, and the political economy of public health. Int J Epidemiol 33(4): 650-667

27. Kouvonen A, Kivimaki M, Vahtera J et al (2006): Psychometric evaluation of a short measure of social capital at work. BMC Public Health 6:251

28. Islam MK, Merlo J, Kawachi I, Lindstrom M \& Gerdtham UG (2006): Social capital and health: does egalitarianism matter? A literature review. Int J Equity Health 5:3

29. International Social Survey Programme (2017):GESIS-Variable Reports No. 2017/23 ISSP 2015 Work Orientations IV Variable Report.

https://www.gesis.org/en/issp/modules/issp-modules-bytopic/work-orientations/2015 (November 20, 2020)

30. Liukkonen V, Virtanen P, Kivimaki M, Pentti J \& Vahtera J (2004): Social capital in working life and the health of employees. Soc Sci Med 59(12): 2447-2458

31. Fujino Y, Kubo T, Kunimoto M et al (2013): A crosssectional study of workplace social capital and blood pressure: a multilevel analysis at Japanese manufacturing companies. BMJ Open 3(2): e002215

32. Suzuki E, Takao S, Subramanian SV, Komatsu H, Doi H \& Kawachi I (2010): Does low workplace social capital have detrimental effect on workers' health? Soc Sci Med 70(9): 1367-1372

33. Chapman LS (2005): Presenteeism and its role in worksite health promotion. Am J Health Promot 19(4): suppl 1-8

34. van den Heuvel SG, Geuskens GA, Hooftman WE,
Koppes LL \& van den Bossche SN (2010): Productivity loss at work; health-related and work-related factors. J Occup Rehabil 20(3): 331-339

35. Nagata T, Mori K, Ohtani M et al (2018): Total healthrelated costs due to absenteeism, presenteeism, and medical and pharmaceutical expenses in Japanese employers. J Occup Environ Med 60(5): e273-e280

36. Johns G (2010): Presenteeism in the workplace: a review and research agenda. J Organ Behav 31(4): 519-542

37. Aronsson G, Gustafsson K \& Dallner M (2000): Sick but yet at work. An empirical study of sickness presenteeism. J Epidemiol Community Health 54(7): 502-509

38. Loeppke R, Taitel M, Haufle V, Parry T, Kessler RC \& Jinnett K (2009): Health and productivity as a business strategy: a multiemployer study. J Occup Environ Med 51(4): 411-428

39. McGregor A, Ashbury F, Caputi P \& Iverson D (2018): A preliminary investigation of health and work-environment factors on presenteeism in the workplace. J Occup Environ Med 60(12): e671-e678

40. Mori K, Mori T, Nagata T et al (2019): Factors of occurrence and improvement methods of presenteeism attributed to diabetes: a systematic review. J Occup Health 61(1): 36-53

41. Goettler A, Grosse A \& Sonntag D (2017): Productivity loss due to overweight and obesity: a systematic review of indirect costs. BMJ Open 7(10): e014632

42. Makishima M, Fujino Y, Kubo T et al (2018): Validity and responsiveness of the work functioning impairment scale (WFun) in workers with pain due to musculoskeletal disorders. J Occup Health 60(2): 156-162

43. Loeppke R, Taitel M, Richling D et al (2007): Health and productivity as a business strategy. J Occup Environ Med 49(7): 712-721

44. Hilton MF, Scuffham PA, Sheridan J, Cleary CM \& Whiteford HA (2008): Mental ill-health and the differential effect of employee type on absenteeism and presenteeism. J Occup Environ Med 50(11): 1228-1243

45. Johnston DA, Harvey SB, Glozier N, Calvo RA, Christensen H \& Deady M (2019): The relationship between depression symptoms, absenteeism and presenteeism. J Affect Disord 256: 536-540

46. Furuichi W, Shimura A, Miyama H et al (2020): Effects of job stressors, stress response, and sleep disturbance on presenteeism in office workers. Neuropsychiatr Dis Treat 16: 1827-1833 
47. Blank N \& Diderichsen F (1995): Short-term and longterm sick-leave in Sweden: relationships with social circumstances, working conditions and gender. Scand J Soc Med 23(4): 265-272

48. Suzuki T, Miyaki K, Song Y et al (2015): Relationship between sickness presenteeism (WHO-HPQ) with depression and sickness absence due to mental disease in a cohort of Japanese workers. J Affect Disord 180: 14-20

49. Ohta M, Higuchi Y, Kumashiro M, Yamato H \& Sugimura H (2017): Decrease in Work Ability Index and sickness absence during the following year: a twoyear follow-up study. Int Arch Occup Environ Health 90(8): 883-894

50. Zhu Y, Gao J, Wang J et al (2018): Association between workplace social capital and absolute presenteeism: a multilevel study in a Chinese context. J Occup Environ Med 60(10): e543-e547

51. van Scheppingen AR, de Vroome EM, ten Have KC, Bos EH, Zwetsloot GI \& van Mechelen W (2013): The associations between organizational social capital, perceived health, and employees' performance in two Dutch companies. J Occup Environ Med 55(4): 371-377

52. Török E, Clark AJ, Jensen JH et al (2018): Work-unit social capital and long-term sickness absence: a prospective cohort study of 32053 hospital employees. Occup Environ Med 75(9): 623-629

53. Rugulies R, Hasle P, Pejtersen JH, Aust B \& Bjorner JB (2016): Workplace social capital and risk of long-term sickness absence. Are associations modified by occupational grade? Eur J Public Health 26(2): 328-333

54. Clausen T, Meng A \& Borg V (2020): Is work group social capital associated with sickness absence? A study of workplace registered sickness absence at the work group level. Saf Health Work 11(2): 228-234

55. Hansen AK, Madsen IEH, Thorsen SV et al (2018): Does workplace social capital protect against longterm sickness absence? Linking workplace aggregated social capital to sickness absence registry data. Scand J Public Health 46(3): 290-296
56. Kessler RC, Barker PR, Colpe LJ et al (2003): Screening for serious mental illness in the general population. Arch Gen Psychiatry 60(2): 184-189

57. Odagiri Y, Ohya Y, Inoue S et al (2010): Reliablity and validation of the Japanese version of the measure of workplace social capital scale. Sangyo Eiseigaku Zasshi 52 (Suppl): 631 (in Japanese)

58. Fujino Y, Uehara M, Izumi H et al (2015): Development and validity of a work functioning impairment scale based on the Rasch model among Japanese workers. J Occup Health 57(6): 521-531

59. Ferrie JE, Kivimaki M, Head J, Shipley MJ, Vahtera J \& Marmot MG (2005): A comparison of self-reported sickness absence with absences recorded in employers' registers: evidence from the Whitehall II study. Occup Environ Med 62(2): 74-79

60. Oke A, Braithwaite P \& Antai D (2016): Sickness absence and precarious employment: a comparative cross-national study of Denmark, Finland, Sweden, and Norway. Int J Occup Environ Med 7(3): 125-147

61. Furukawa TA, Kawakami N, Saitoh M et al (2008): The performance of the Japanese version of the K6 and K10 in the World Mental Health Survey Japan. Int J Methods Psychiatr Res 17(3): 152-158

62. Sakurai K, Nishi A, Kondo K, Yanagida K \& Kawakami N (2011): Screening performance of K6/K10 and other screening instruments for mood and anxiety disorders in Japan. Psychiatry and clinical neurosciences 65(5): 434-441

63. Ferlander S (2007): The importance of different forms of social capital for health. Acta Sociologica 50(2): 115-128

64. Knapstad M, Holmgren K, Hensing G \& Overland S (2014): Previous sickness absence and current low perceived social support at work among employees in the general population: a historical cohort study. BMJ Open 4(10): e005963

J UOEH $43(3): 293-303(2021)$ 